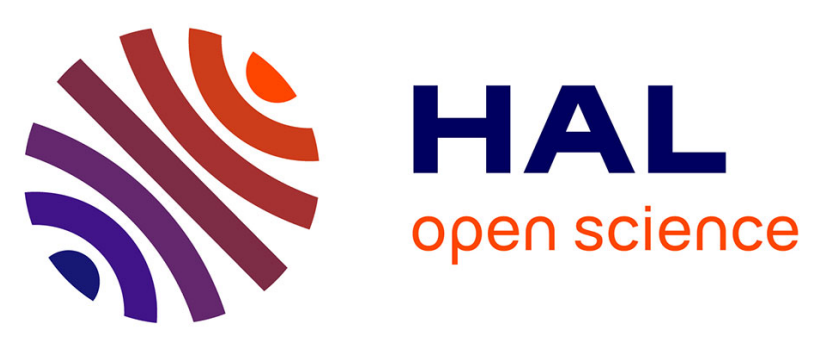

\title{
In vivo Efficacy of a Dry Powder Formulation of Ciprofloxacin-Copper Complex in a Chronic Lung Infection Model of Bioluminescent Pseudomonas aeruginosa
}

Frederic Tewes, Tania F Bahamondez-Canas, Daniel Moraga-Espinoza, Hugh

D C Smyth, Alan B Watts

\section{To cite this version:}

Frederic Tewes, Tania F Bahamondez-Canas, Daniel Moraga-Espinoza, Hugh D C Smyth, Alan B Watts. In vivo Efficacy of a Dry Powder Formulation of Ciprofloxacin-Copper Complex in a Chronic Lung Infection Model of Bioluminescent Pseudomonas aeruginosa. European Journal of Pharmaceutics and Biopharmaceutics, 2020, 152, pp.201-217. 10.1016/j.ejpb.2020.05.014 . inserm-02615177

\section{HAL Id: inserm-02615177 https://www.hal.inserm.fr/inserm-02615177}

Submitted on 22 May 2020

HAL is a multi-disciplinary open access archive for the deposit and dissemination of scientific research documents, whether they are published or not. The documents may come from teaching and research institutions in France or abroad, or from public or private research centers.
L'archive ouverte pluridisciplinaire HAL, est destinée au dépôt et à la diffusion de documents scientifiques de niveau recherche, publiés ou non, émanant des établissements d'enseignement et de recherche français ou étrangers, des laboratoires publics ou privés. 


\section{In vivo Efficacy of a Dry Powder Formulation of Ciprofloxacin-Copper Complex in a Chronic Lung Infection Model of Bioluminescent Pseudomonas aeruginosa}

Frederic Tewes ${ }^{1,2, *}$, Tania F. Bahamondez-Canas ${ }^{2,3,4}$, Daniel Moraga-Espinoza ${ }^{2,3,4}$, Hugh D.C. Smyth², Alan B. Watts ${ }^{2}$

${ }^{1}$ INSERM U1070, UFR de Médecine Pharmacie, Université de Poitiers, 1 rue Georges Bonnet, TSA 51106, 86073 Poitiers Cedex 9, France

${ }^{2}$ College of Pharmacy, The University of Texas at Austin, 2409 West University Avenue, PHR 4.214, Austin, TX 78712, USA

${ }^{3}$ Escuela de Farmacia, Universidad de Valparaiso, Gran Bretaña 1093, Playa Ancha, Valparaíso, Chile

${ }^{4}$ Centro de Investigación Farmacopea, Universidad de Valparaíso, Santa Marta 183, Playa Ancha, Valparaíso, Chile

* Corresponding author: ftewes@univ-poitiers.fr 


\section{AbStraCt}

A significant limitation of locally delivered treatments for chronic pulmonary infections is often the short residence time within the airways. Ciprofloxacin (CIP), for example, undergoes rapid absorption from the airway lumen. Previously, we demonstrated that the complexation of CIP with copper $(\mathrm{CIP}-\mathrm{Cu})$ reduces its apparent epithelial permeability and pulmonary absorption rate without affecting antimicrobial activity against Pseudomonas aeruginosa grown planktonically or as biofilms. This study aimed to evaluate the in vivo efficacy of $\mathrm{CIP}-\mathrm{Cu}$, prepared as a dry powder, in a chronic lung infection model. The powders were prepared by jet milling $(\mathrm{CIP}-\mathrm{HCl})$ and by spray drying $(\mathrm{CIP}-\mathrm{Cu})$. A bioluminescent strain of $P$. aeruginosa (PAO1::p16Slux) was used to prepare bacteria-loaded agar beads that were inoculated intratracheally to rats. The dynamics of the infection were monitored using luminometry. The bacteria/beads ratio was optimized to allow the highest luminescence signal and animal survival for 8 days. The efficacy of the treatment was evaluated by luminometry in addition to the endpoint (Day 8) where colony counting was performed after lung harvesting. Luminescent $P$. aeruginosa entrapped in agar beads were useful to monitor the spatial development of the chronic lung infection in rats. The rats were treated with the dry powders in a nose-only inhalation exposure system (NOIES). CIP-Cu and CIP-HCl powders showed similar aerodynamic properties and comparable CIP lung deposition. However, treatment with CIP-Cu significantly $(\mathrm{p}<0.01)$ reduced by $4-\log$ the number of CFU of $P$. aeruginosa per lung in the chronic infection model, whereas CIP-HCl effect was not different from the untreated control group. 


\section{INTRODUCTION}

Effective therapy using pulmonary delivery of antibiotics can be limited by the short drug residence time at the site of infection, but a novel complexed form of ciprofloxacin may help overcome this obstacle while maintaining antimicrobial activity against resistant biofilm bacterial infections $[1,2]$. Pulmonary delivery of antibiotics in solution form has demonstrated clinical benefit in the treatment of chronic bacterial lung infections, as it can allow for significantly higher drug concentrations at the site of infection with reduced systemic levels compared to i.v. administration [3]. This is mainly true for antibiotics having very low lungblood barrier permeability, such as the currently used tobramycin, colistin methanesulfonate and aztreonam lysine. However, this approach is less suitable for molecules that readily diffuse through the lung epithelial barrier and rapidly equilibrate with blood concentrations, such as in the case of ciprofloxacin (CIP) [4-7]. Thus, for antimicrobial molecules having relatively high permeability across the lung-blood barrier, advanced formulations can be developed to retain the drug in the airways by slowing down the drug flux across this barrier. These advanced formulations generally act either by reducing and sustaining the drug release rate from formulation [8-13], or by reducing the apparent permeability of the drug across the epithelium [2, 14-17]. In order to increase CIP lung residency time after its aerosolization, we recently developed a novel positively charged complex made of CIP and copper $[2,14,18]$. This CIPcopper complex (CIP-Cu) allowed a $90 \%$ decrease in the apparent permeability of CIP across a Calu-3 cell line model of pulmonary epithelium $[2,18]$. This decrease in permeability translated in vivo as a decrease in the lung-blood absorption rate of CIP and a sustained high CIP concentration in the lung epithelial lining fluid (ELF) after intratracheal delivery to rats [2]. Additionally, the in vitro antibacterial activities of CIP and CIP-Cu evaluated against $P$. 
aeruginosa grown in planktonic cultures $[14,18]$ or as biofilms [1] were also found to be equivalent.

The aim of this study was, therefore, to evaluate the in-vivo efficacy of CIP-Cu complexloaded particles after pulmonary inhalation in model rats that developed chronic pulmonary infection by instillation of Pseudomonas aeruginosa-loaded agar beads $[19,20]$. This approach has been shown to model the chronic infection and inflammation that affect cystic fibrosis patients $[19,21,22]$. In order to assess temporal effects of the treatments on the infection, we also evaluated time resolved luminescence of a bioluminescent $P$. aeruginosa, PAOI strain to non-invasively monitor the chronic lung infections in rats. 


\section{MATERIALS AND METHODS}

\subsection{Materials}

The bioluminescent PAO1::p16Slux, a PAO1 strain (gift from Professor Shawn Lewenza from the university of Calgary, Canada) tagged by chromosomal integration of p16Slux construction [23], was used to prepare bacteria-loaded agar beads and monitor the chronic lung infection. Ciprofloxacin base (CIP) powder (purity $\geq 98,0 \%$ ), copper hydroxide $\mathrm{Cu}(\mathrm{OH})_{2}$, hyaluronic acid (HA) sodium salt from Streptococcus equi, calcium hydroxide $\mathrm{Ca}(\mathrm{OH})_{2}$, formic acid and ammonium carbonate $\left(\mathrm{NH}_{4}\right)_{2} \mathrm{CO}_{3}$ were purchased from Sigma Aldrich (St Louis, USA). Ciprofloxacin hydrochloride monohydrate USP grade was purchased from Letco Medical (Decatur, AL, USA)

\subsection{Preparation and characterization of the dry powder formulations}

\subsubsection{Preparation of ciprofloxacin-copper loaded microparticles}

Microparticles composed of amorphous calcium carbonate and CIP-Cu complex (Spray dried CIP-Cu) were prepared by spray-drying as previously described [14]. Briefly, two aqueous solutions were prepared. One solution was made of CIP, calcium hydroxide, sodium hyaluronate, formic acid, and copper hydroxide. The other one was made of ammonium carbonate. These solutions were prepared separately and mixed during the microparticle preparation process using a Y-tube connected to the feeding tube of the B-290 mini spray dryer (Büchi, Labortechnik AG, Switzerland). The spray dryer was operated in open mode with $27 \mathrm{~mL} / \mathrm{min}$ peristaltic pump rate, $15 \mathrm{~L} /$ min spraying air flow rate, $630 \mathrm{~L} / \mathrm{h}$ drying air flow rate, and $120^{\circ} \mathrm{C}$ inlet temperature. Spray dried CIP-Cu powder was stored at $4^{\circ} \mathrm{C}$ in desiccated condition. 


\subsubsection{Preparation of jet milled ciprofloxacin powder}

Microparticles made of ciprofloxacin hydrochloride (Micronized $\mathrm{CIP}-\mathrm{HCl}$ ) were micronized by a jet mill Model 00 Jet-O-Mizer ${ }^{\mathrm{TM}}$ (Fluid Energy, Telford, PA, USA). For some drugs, this method can produce an easily aerosolizable powder without the use of excipients [24]. Micronized CIP-HCl powder was stored at room temperature in desiccated condition.

\subsubsection{Scanning electron microscopy}

Samples mounted on standard aluminum SEM stubs were sputter coated with $12 \mathrm{~nm}$ platinum/palladium $(\mathrm{Pt} / \mathrm{Pd})$ in an argon atmosphere using a sputter coater 208HR (Cressington Scientific Instruments Ltd., Watford, UK). The particles were imaged using a Zeiss Supra 40VP SEM (Carl Zeiss Microscopy GmbH, Jena, Germany).

\subsubsection{Particle size distribution analysis}

Particle size distributions (PSD) were determined by laser diffraction using the Sympatec HELOS-R and a dry powder disperser (RODOS - Sympatec GmbH, Clausthal-Zellerfeld, Germany). RODOS was loaded with approximately $3 \mathrm{mg}$ of powder and used at different dispersion pressures in a range of 0.5-4.0 Bar. PSD was calculated using the Fraunhofer approximation $(\mathrm{n}=3)$.

\subsubsection{Ciprofloxacin assay}

CIP was assayed by measuring the CIP fluorescence using a plate reader (Infinite M200, Tecan Systems Inc., CA, USA). CIP standards (concentration ranging from 3.75 to $0.03 \mu \mathrm{g} / \mathrm{mL}$ ), controls $(3,0.5$ and $0.06 \mu \mathrm{g} / \mathrm{mL})$ and samples were half-diluted in $0.5 \mathrm{M}$ sodium acetate buffer $\mathrm{pH} 4.5$, containing $0.05 \mathrm{M}$ of EDTA to complex the metal cation that could interact with CIP and 
modify its spectroscopic properties. The maximum excitation wavelength of CIP was $302 \mathrm{~nm}$ and its maximum fluorescence emission wavelength was $456 \mathrm{~nm}$.

\subsection{Preparation and characterization of $P$. aeruginosa-loaded agar beads}

\subsubsection{Preparation of the P. aeruginosa-loaded beads}

Sterile and bacteria-loaded agar beads were prepared using an adapted version of the method described by Growcott et al. [22]. Fresh suspension of bioluminescent P. aeruginosa (strain PAO1::p16Slux) prepared in BHI broth was cultured to exponential growth phase and then adjusted to an $\mathrm{OD}_{600}$ of 0.3 . Two milliliters of this suspension were washed and concentrated in $1 \mathrm{~mL}$ of PBS by centrifugation. The bacteria were embedded into agar beads by mixing $1 \mathrm{~mL}$ of this concentrated suspension with $9 \mathrm{~mL}$ of molten $2 \% \mathrm{w} / \mathrm{v} \mathrm{LB}$ agar (Fisher Scientific, NJ, USA). The mixture was then emulsified into $100 \mathrm{~mL}$ of warmed $\left(48{ }^{\circ} \mathrm{C}\right)$ paraffin oil (Sigma Aldrich, USA) containing $0.01 \% \mathrm{v} / \mathrm{v}$ of sorbitan monooleate $\left(\mathrm{SPAN}^{\circledR} 80\right.$, Sigma Aldrich, USA) based on the report of Growcott et al. [22]. The emulsion was cooled, the beads centrifuged at 10,000 $\mathrm{g}$ for $10 \mathrm{~min}$ and then 4-times washed with PBS pH 7.4. Ten milliliters of the bead suspension in PBS were stored up to 2 weeks at $4^{\circ} \mathrm{C}$. For sterile agar beads, the volume of bacterial suspension was replaced with PBS.

The number of bacteria per bead was determined from a CFU count performed with 100 $\mu \mathrm{L}$ of the bead suspension diluted in $5 \mathrm{~mL}$ of PBS and homogenized for 1 min (Polytron PT2100, Kinematica AG, Switzerland). Ten-fold serial dilutions of this suspension were spread on LB agar plates (Hardy Diagbostics, CA, USA) for colony counts. Bead concentrations were determined using a hemocytometer that allowed calculating the number of bacteria per beads.

Size distribution of blank beads was determined by laser diffraction using the Sympatec HELOS-R equipped with a Cuvette module (Sympatec GmbH, Clausthal-Zellerfeld, Germany). 
A reference measurement was performed on the cell filled with $50 \mathrm{~mL}$ of PBS. After the reference measurement, an aliquot of sterile beads suspension was added to the cell to achieve optical concentrations between $10-20 \%(n=3)$.

\subsubsection{Time-luminescence intensity kinetics of bacteria-loaded agar beads}

In vitro behavior of the luminescent $P$. aeruginosa in the agar beads was evaluated by following the luminescence produced by the bacteria within the beads versus time in the presence of various $\mathrm{CIP}$ or $\mathrm{CIP}-\mathrm{Cu}$ complex concentrations. One hundred and eighty microliters

of agar bead suspension at $1.5 \times 10^{5}$ beads $/ \mathrm{mL}$ and containing 25 bacteria/bead were added into the wells of a black 96-well plate with flat clear bottom (Corning Inc. NC, US). Wells were supplemented with $20 \mu \mathrm{L}$ of 10 -times concentrated two-fold dilution of CIP or CIP-Cu complex in BHI. Final CIP concentrations ranged from 64 to $0.062 \mu \mathrm{g} / \mathrm{mL}$. Then, the plates were covered using an air-permeable membrane and incubated at $37{ }^{\circ} \mathrm{C}$ in the plate reader (SpectraMax M3, Molecular Devices LLC, CA, USA). Luminescence intensity emitted from the bottom of the wells was measured during 1 second per well every 20 min. Plates were shaken before each measurement $(\mathrm{n}=3)$.

\subsection{Development of the chronic lung infection model}

\subsubsection{Animal model}

The protocol for the animal study was approved by the Institutional Animal Care and Use Committee (IACUC) at the University of Texas at Austin, Austin, TX. Sprague-Dawley rats weighting between 225 and $275 \mathrm{~g}$ were purchased from Charles River Laboratories (Wilmington, MA). Animals were housed one per cage, subjected to $12 \mathrm{~h} / 12 \mathrm{~h}$ light and darkness cycles with access to food and water ad libitum. 


\subsubsection{Inoculation of rats with bioluminescent $P$. aeruginosa-loaded agar beads}

On day 1 , anesthetized (Ketamine/xylazine $(80 / 6 \mathrm{mg} / \mathrm{Kg})$ i. p.) male Sprague-Dawley rats were inoculated with various dose of $P$. aeruginosa ( 0 to $\left.5 \times 10^{7} \mathrm{CFU}\right)$ embedded into agar beads. Intratracheal (i.t.) inoculation was performed by instilling $100 \mu \mathrm{L}$ of agar bead suspension loaded in a syringe connected to a dry powder insufflator cannula (Penn-Century Inc, Philadelphia, USA). This cannula has a blunt-tip designed to pass between the vocal cords without cutting blood vessels or damaging the epithelium. The vocal cords were visualized using a special laryngoscope suitable for rodent (Penn-Century Inc, Philadelphia, USA). Animals were kept on a warming pad until full recovery from anesthesia. Body weight and luminescence emitted from the rats anesthetized with isoflurane were measured daily for 8 days using an in vivo imaging system IVIS ${ }^{\circledR}$ Spectrum (PerkinElmer, Houston, TX, USA) with an exposure time of 3 min per rat.

\subsection{In vivo evaluation of ciprofloxacin-copper inhalation treatment}

\subsubsection{Pulmonary dosing and regimen}

A nose-only inhalation exposure system (NOIES) capable of dosing 24 rats at a time was used (CH Technologies (USA) Inc., Westwood, NJ, USA). Prior to dosing, the animals were individually acclimated for 30 min per day for four days in restraint tubes. Animals were dosed on day 4 and day 6 post infections (Figure 1).

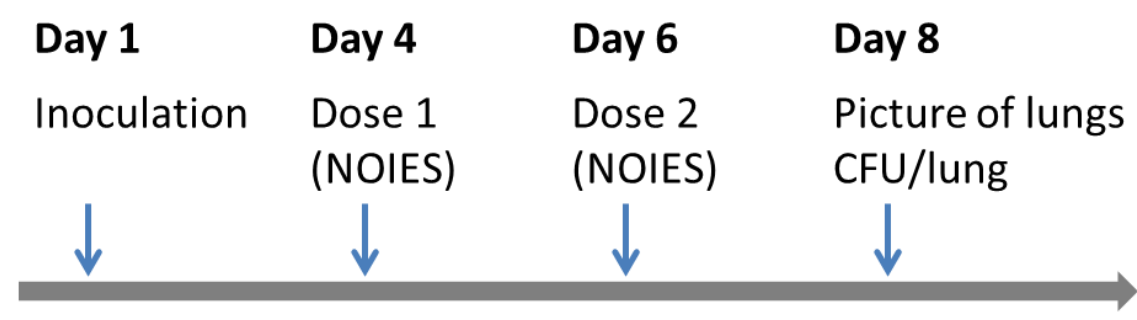

Average radiance (IVIS)

Body weight (g) 
Figure 1: Dosing regimen after i.t. inoculation of agar beads loaded with bioluminescent PA01. Ciprofloxacin doses were set at $1.65 \mathrm{mg}$ of CIP (base) per animal.

Powders for inhalation were loaded into a feed cylinder connected to a rotating brush aerosol generator (RGB 1000). At an airflow rate of $0.5 \mathrm{~L} / \mathrm{min}$, animals treated with spray dried CIP-Cu were dosed for $35 \mathrm{~min}$ and animals treated with micronized CIP-HCl were dosed for 20 min, so that equivalent doses of CIP $(1.65 \mathrm{mg} /$ animal $)$ were given. The purpose of the $0.5 \mathrm{~L} / \mathrm{min}$ flow was to transport the aerosolized particles to the animal at a flow slightly higher than the minute ventilation of a rat. Following dosing, animals were placed back into their respective cages. These times of exposures were calculated to provide the same Total inhaled dose (TID mg) by rats and determined according to Eq. (1) as described by Alexander et al. [25].

$$
T I D=C \times R M V \times D \times P
$$

where $C$ is the measured particle concentration in air $(\mathrm{mg} / \mathrm{L}), R M V$ is the species-specific respiratory minute volume or the volume of air inhaled in one minute per animal $(\mathrm{L} / \mathrm{min}), D$ is the duration of exposure (min), and $P$ is the particle drug potency (mass of CIP base per mass of particles -0.4 for $\mathrm{CIP}-\mathrm{Cu}$ and 0.86 for $\mathrm{CIP} \cdot \mathrm{HCl}$ ). The $\mathrm{RMV}$ for rats was calculated according to the Eq. (2) [25].

$$
R M V=0.608 \times B W^{0.852}
$$

where $B W(\mathrm{~kg})$ is the body weight of the rats. The aerosol concentration in air $C$, was calculated based on the average mass of samples deposited on filters $(n=3)$ taken from the NOIES inhalation chamber throughout the exposure using a vacuum flow of $0.5 \mathrm{~L} / \mathrm{min}$. To determine the portion of the TID that was deposited in the lungs, the percent deposition in relationship to the aerodynamic PSD was estimated based on rodent dosimetry findings reported by Kuehl et al. [26]. Thus, a Mercer-style mini cascade impactor (CH Technologies (USA) Inc., Westwood, NJ, USA) was used to determine the aerodynamic PSD of both aerosolized powders present 
within the nose-only exposure chamber. This impactor is a seven-stage cascade impactor that has been manufactured to be plugged directly to the nose-only exposure system, in exactly the same position as the animal restraint tubes used to connect the animal to the inhalation chamber $[26$, 27]. The particles were collected at a rate of $0.5 \mathrm{~L} / \mathrm{min}$ on this seven impactor stages having the following cut-off diameters at this specific airflow rate $(5.14,3.04,1.89,1.16,0.77,0.48,0.35$ $\mu \mathrm{m})$. The MMADs were determined by differential weight analysis of the stages before and after impaction using a microbalance (METTLER TOLEDO, Columbus, OH, USA) and plotting the inverse of the standard normal cumulative mass distribution less than stated size cut-off against the natural logarithm of the cut-off diameter of the respective stages. The MMAD of the particles distribution was defined as the particle size at which the line crosses the $50 \%$ mark.

\subsubsection{Lung harvest for microbiological assessment}

Eight days after infection, the animals in each group were euthanized by $\mathrm{CO}_{2}$ inhalation, right after measuring the luminescence. Animals were cleaned with $70 \% \mathrm{v} / \mathrm{v}$ ethanol solution before lungs collection. Lungs were homogenized in PBS and serial dilutions of these homogenates were plated onto LB agar plates to measure the number of CFU per lung. ( $n=4$ for the untreated group; $\mathrm{n}=4$ for the CIP-HCl group, and $\mathrm{n}=7$ for the $\mathrm{CIP}-\mathrm{Cu}$ group)

\subsubsection{Statistical analysis}

Statistical analyses were performed using GraphPad Prism 6.0 (GraphPad Software Inc.). All data were expressed as means $\pm \mathrm{SD}$ and analysed with the Dunn's Kruskal-Wallis multiple comparisons test. $p<0.05$ was considered statistical significance. 


\section{RESUltS}

\subsection{Preparation and characterization of the dry powder formulations}

The SEM micrographs of spray dried CIP-Cu and micronised CIP-HCl powders showed different morphologies (Figure 2A). Spray dried CIP-Cu particles were spherical hollow brittle particles while jet milled CIP-HCl particles were solid and non-spherical. The RODOS dry powder disperser was used to compare the dispersibility of the powders at different pressures (Figure 2B). The parameters $\mathrm{D}_{10}, \mathrm{D}_{50}$ and $\mathrm{D}_{90}$, represent the $10 \%, 50 \%$ and $90 \%$ point in the cumulative undersize PSD, respectively. The increase in the dispersion pressure decreased mainly the $\mathrm{D}_{90}$ and $\mathrm{D}_{50}$ of the powders. This behavior was more noticeable for the spray dried CIP-Cu powder than for the jet milled CIP-HCl. However, both powders had similar PSD when they were dispersed with a pressure of 3 bar or above.

A

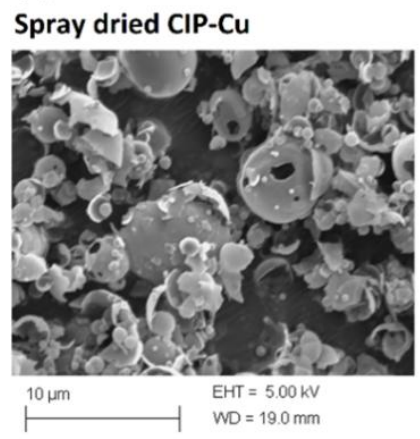

Jet milled CIP-HCI

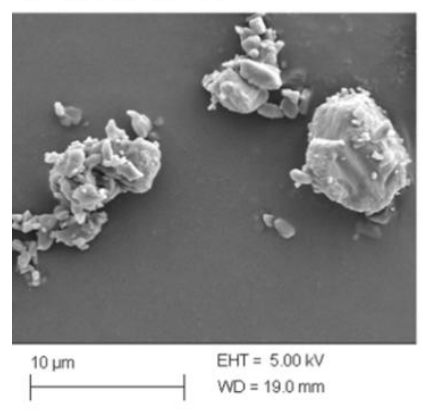

B

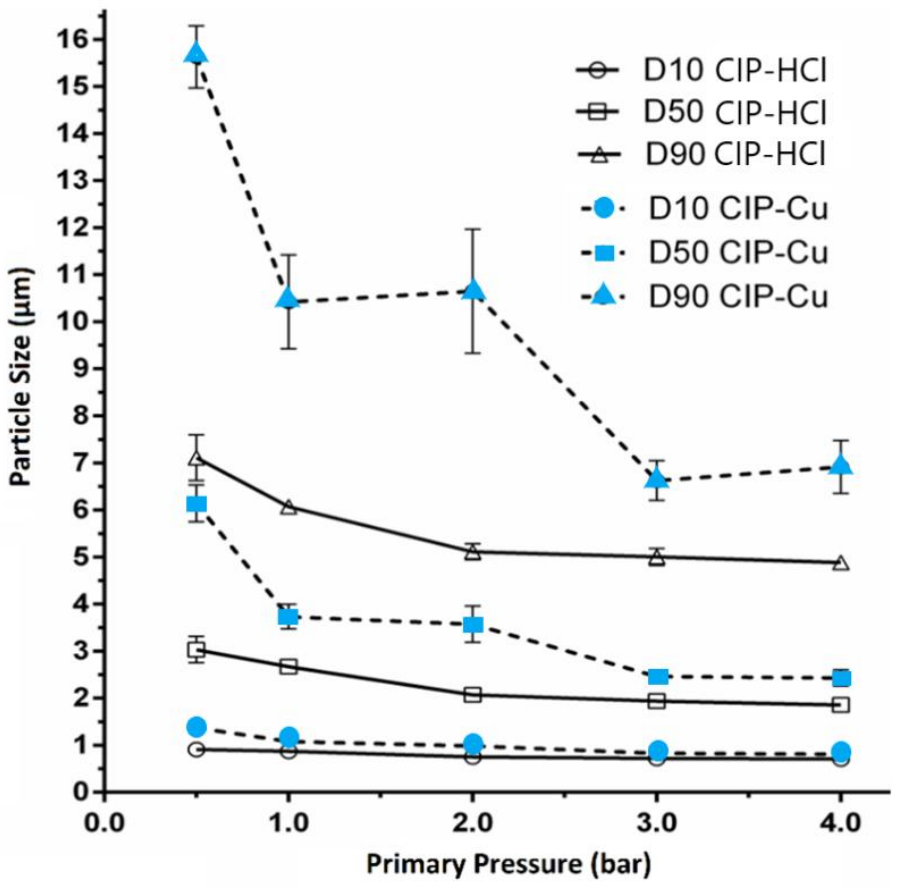

Figure 2: Characterization of spray dried CIP-Cu and jet milled CIP-HCl powders. A. SEM micrographs, B. Evolution of the PSD parameters $D_{10}, D_{50}$, and $D_{90}$ versus dispersion pressure. Solid symbols are for spray dried CIP-Cu, and open symbols are for micronized CIP-HCl. Values are means \pm SD $(n=3)$. 


\subsection{Characterization of the $P$. aeruginosa-loaded agar beads}

The geometric size distribution (weighed in volume) of the sterile agar beads was monodisperse with a median value of $88 \pm 3 \mu \mathrm{m}$ as indicated by the $\mathrm{D}_{50}$ (Figure 3 ).

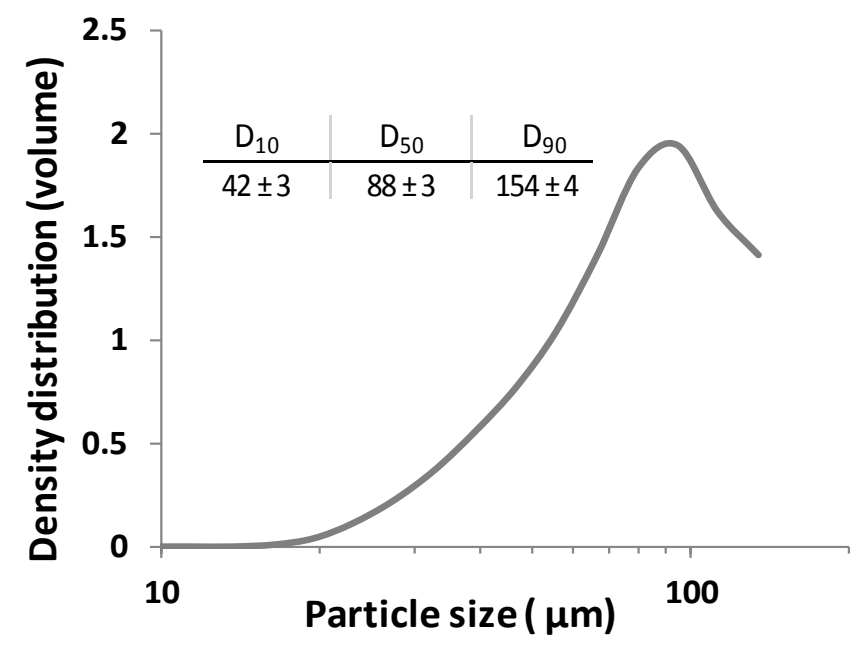

Figure 3: Size distribution of agar beads dispersed in PBS. Size distribution of blank beads was determined by laser diffraction using the Sympatec HELOS-R equipped with a Cuvette module. Values are means \pm SD $(n=3)$.

Prior to animals inoculation, we evaluated the in vitro susceptibility of $P$. aeruginosa embedded in the agar beads to CIP-HCl and the CIP-Cu complex by recording bioluminescence curves over time in the presence of various equivalent concentrations of CIP base (Figure 4). Overall, CIP-HCl and CIP-Cu induced similar effect on the bacteria proliferation profiles. For concentrations above $0.5 \mu \mathrm{g} / \mathrm{mL}$ and up to $32 \mu \mathrm{g} / \mathrm{mL}$, both treatments induced a continuous decrease in bacterial luminescence. Interestingly, for concentration between 0.063 and 0.25 $\mu \mathrm{g} / \mathrm{mL}$ (Figure 4, top), the initial decrease in luminescence was followed by an increase up to 15 $-20 \mathrm{~h}$. 

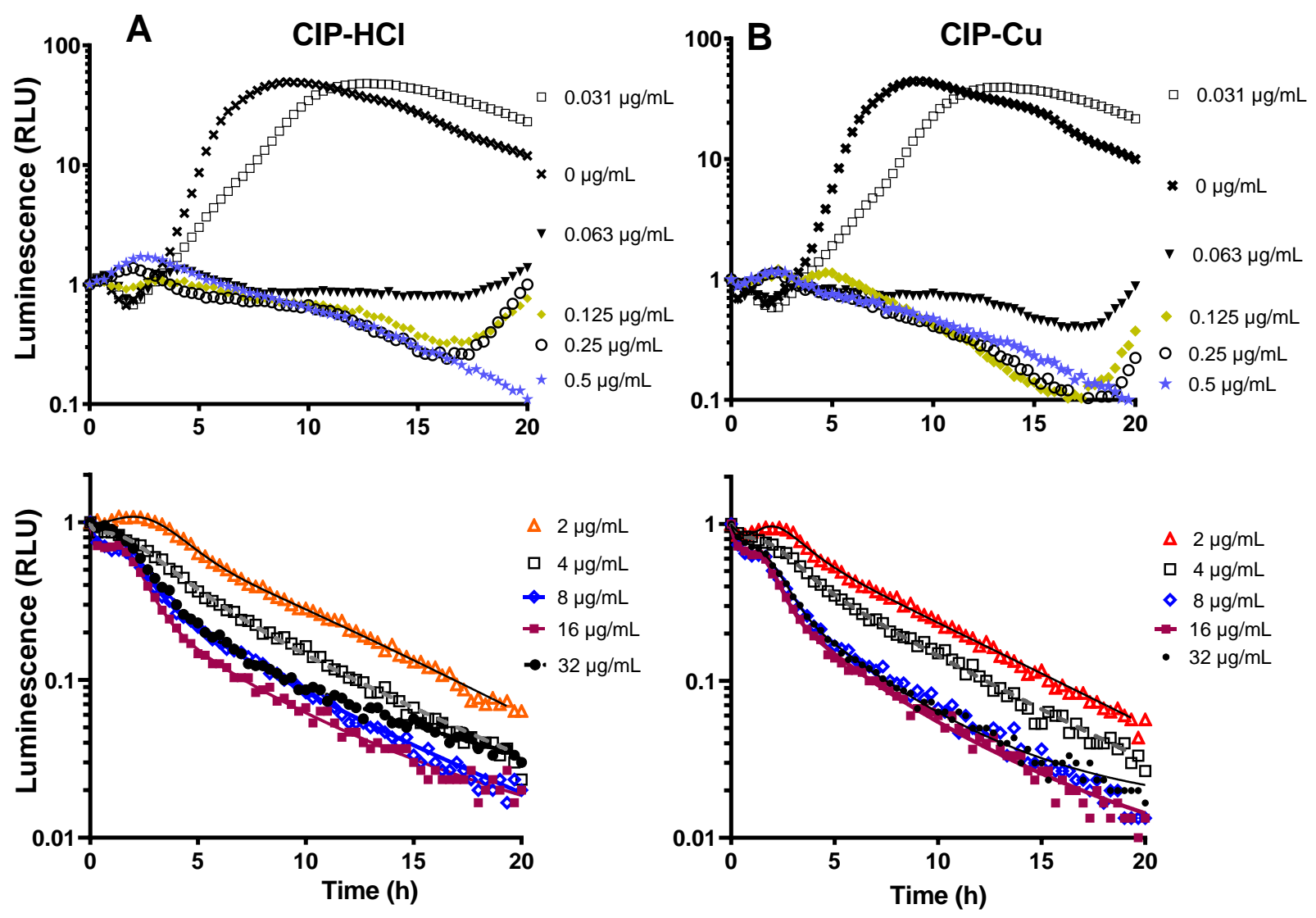

Figure 4: Time-luminescence curves obtained from bioluminescent $P$. aeruginosa-loaded beads. The beads were incubated with different CIP-base equivalent concentrations of CIPHCl (A) and CIP-Cu (B). (Top: $0-0.5 \mu \mathrm{g} / \mathrm{mL}$, bottom: $2-32 \mu \mathrm{g} / \mathrm{mL}$ ). Luminescence (as relative light units (RLU)) was recorded every $20 \mathrm{~min}(\mathrm{n}=3$; mean).

\subsection{Development of the chronic lung infection model with bioluminescent bacteria}

To develop the chronic lung infection model, we evaluated different bacteria/bead ratios for i.t. inoculation of the animals. The luminescence emitted by $P$. aeruginosa from the thorax of the inoculated rats was recorded daily and the average radiance values (photon $/ \mathrm{sec} / \mathrm{cm}^{2} / \mathrm{sr}$ ) were plotted versus time after inoculation (Figure 5A). Just after inoculation (day 1), a large part of the signal was located at the carina as indicated by the luminescence images obtained with IVIS (Figure 5B), and no significant differences in luminescence were observed between the inoculums. On day 2, the signal sharply increased for the three inoculums tested in proportion to CFU numbers of the inoculum. In all cases, the increase in signal on day 2 was associated with a 
loss of the animals' body weight. By day 3, the CIP-HCl group had lost $11.8 \%$ of body weight and the CIP-Cu group had lost $6.9 \%$ relative to average body weight on day 1 . By day 8 , the average body weight of the groups was similar to the body weight on day 1 , with CIP-HCl group reaching $99.7 \%$ of the initial weight and CIP-Cu reaching $97.6 \%$ of the initial body weight.

A

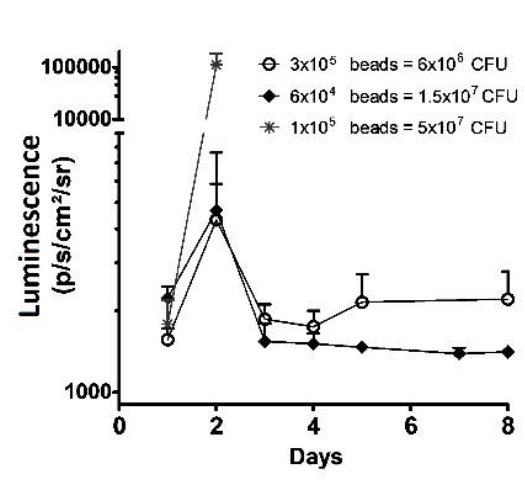

B

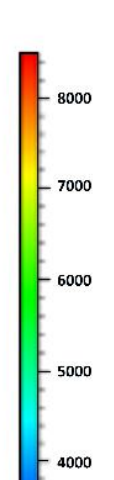

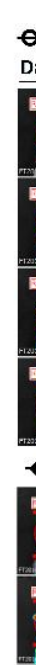

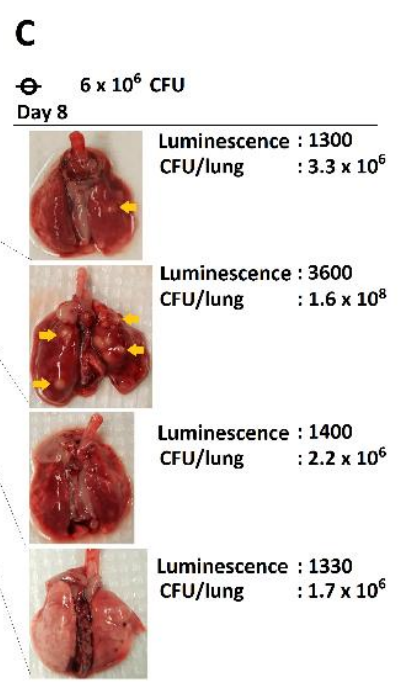

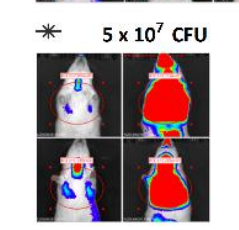

Figure 5: In vivo chronic lung infection model. Sprague-Dawley rats were inoculated intratracheally with various ratios of luminescent $P$. aeruginosa entrapped in agar beads (CFU/beads ratios): 20 CFU/bead $\left(6 \times 1^{6}\right.$ CFU/animal), 250 CFU/bead $\left(\begin{array}{llll}1.5 & \times & 10^{7}\end{array}\right.$ CFU/animal), and $500 \mathrm{CFU} / \mathrm{bead}\left(5 \times 10^{7} \mathrm{CFU} /\right.$ animal). A. Variation of luminescence (in photons $\mathrm{s}^{-1} \mathrm{~cm}^{-2}$ steradian $\left.^{-1}\right)$ per day $(\mathrm{n}=2-4$; mean \pm S.E.M.). B. Luminescence imaging obtained in IVIS. C. Pictures of the infected lungs on day 8 and number of CFU recovered from the lungs (group 6 x $10^{6} \mathrm{CFU} / \mathrm{animal}$ ).

In the highest inoculum ( $5 \times 10^{7} \mathrm{CFU}$; $500 \mathrm{CFU} /$ bead), the signal increased 100-times compared to day 1 , however, none of the animals survived until day 3 . For the 2 lower inoculums $\left(1.5 \times 10^{7}\right.$ and $6 \times 10^{6} \mathrm{CFU} /$ animal), after a sharp luminescence increase on day 2 , the signals decreased on day 3 to values close to these obtained on day 1 and then were stable from day 3 to 
day 8 . The inoculum with $6 \times 10^{6} \mathrm{CFU}(20 \mathrm{CFU} / \mathrm{bead})$ gave the best survival rate associated to the highest and stable signal for 3 days. On day 8 , the lungs of these rats had visible nodules (yellow arrows, Figure 5C) that correlated with values of luminescence and the number of CFU recovered from the lungs after plating. This inoculum was selected to evaluate the in vivo efficacy of the CIP powders after pulmonary inhalation.

\subsection{In vivo evaluation of ciprofloxacin-copper inhalation treatment}

Powders were administered to rats by the inhalation route using the NOIES that allowed dosing multiple animals in a time (Figure 6A). In order to compare the efficacy of the CIP-HCl and CIP$\mathrm{Cu}$ powders in vivo, the same equivalent dose of CIP, with a similar lung deposition, had to be delivered to rats. Therefore, we evaluated the aerodynamic properties of the powders present in the inhalation chamber using a Mercer-Style mini cascade impactor directly connected to the NOIES. Aerodynamic PSD of the powders' particles present in the inhalation chamber are presented in Figure 6B. Both formulations had a narrow PSD and similar MMADs in the 1 to 3 $\mu \mathrm{m}$ range. Particles having an MMAD in this range generally give a same percentage of lung deposition in rats of approximately $20 \%$ as shown by Kuehl et al.[26]. Moreover, both formulation particles in the NOIES inhalation chamber have close GSD indicating a narrow dispersity of the particles from the MMAD value. Therefore, the same lung deposition should occur with both formulations. The other parameters evaluated before dosing the animals were the Total Inhaled Dose (TID) and the time needed to achieve the same TID in each group of animals ( $D$ in Eq. 1). The potency of CIP-Cu was lower than the CIP-HCl due to the presence of more excipients $\left(\mathrm{CaCO}_{3}\right.$ and hyaluronic acid). Thus, animals were dosed for 35 min with $\mathrm{CIP}-\mathrm{Cu}$ and for 20 min with CIP-HCl to achieve comparable TID of around $1.65 \mathrm{mg}$. Using this TID, the aerodynamic properties of the powder particles evaluated with the Mercer-Style mini-cascade 
impactor and the dosimetry results obtained on rats reported by Kuehl et al [26], the estimated lung depositions were calculated and similar values were obtained between the two powders (Figure 6C).
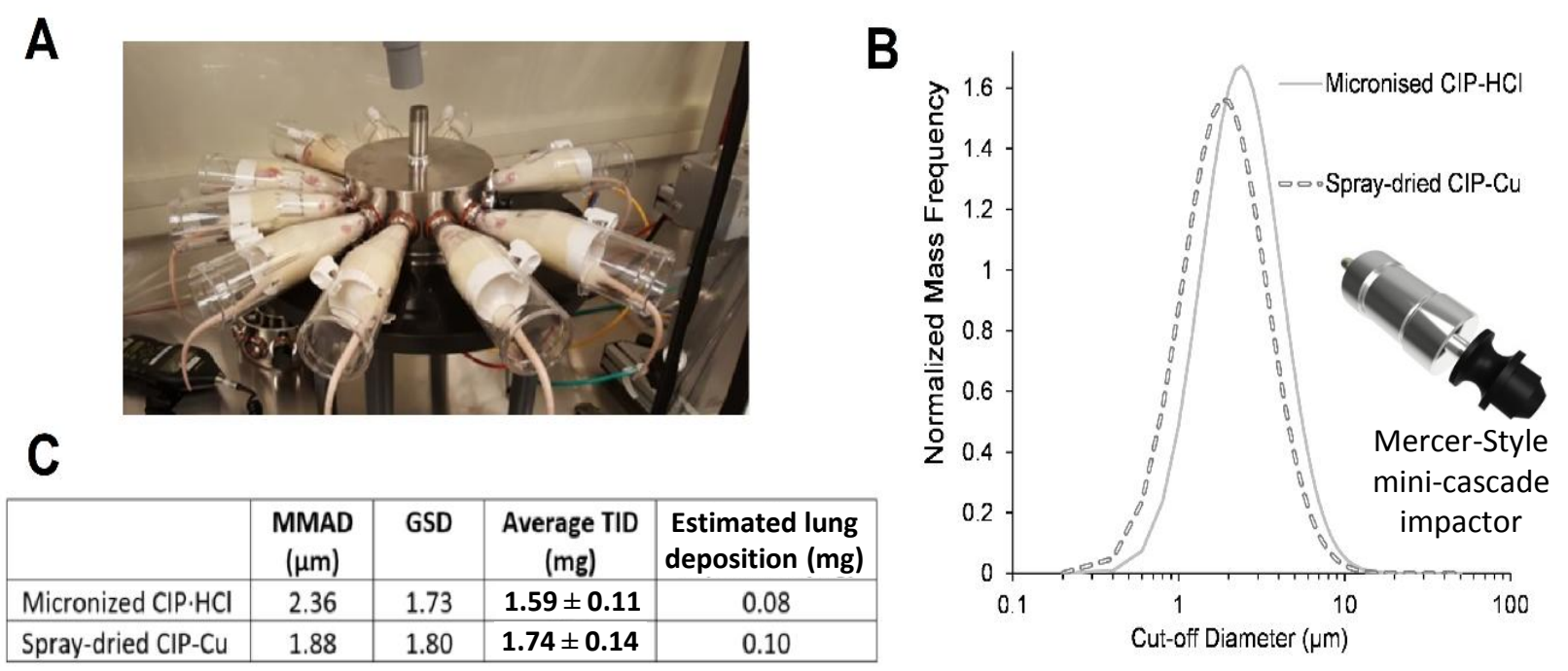

Figure 6: Microparticles aerodynamic properties measured using a Mercer-Style minicascade impactor connected to the nose-only inhalation exposure system (NOEIS). A) Animals treated in NOIES, B) Distribution of the aerodynamic size of the particles present in the NOIES measured using the Mercer-Style mini-cascade impactor (picture inserted). C) Aerodynamic properties parameters (MMAD: Mass median aerodynamic diameter, GSD: Geometric standard deviation, TID: Total Inhaled Dose).

The in vivo efficacy of both formulations was measured by luminometry and by the number of $\mathrm{CFU}$ in the lung (CFU/lung) (Figure 7). Infected animals were dosed with the formulations at day 4 and 6 after infection. Both treatments induced a decrease in luminescence compared to the untreated group, however, due to the variability and small groups, these differences were not statistically significant (Figure 7A). On the other hand, a significant decrease of 4 logs in the number of CFU/lung was obtained with the CIP-Cu compared to the non-treated group $(\mathrm{p}<0.01$; Figure $7 \mathrm{~B})$. In the same condition, CIP-HCl effect was not different from the control group. 


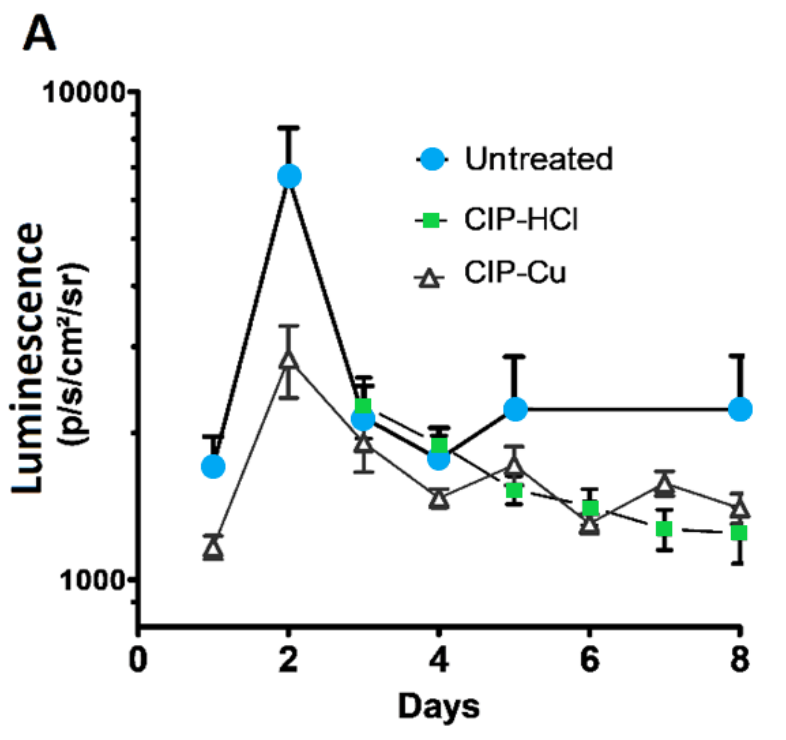

B

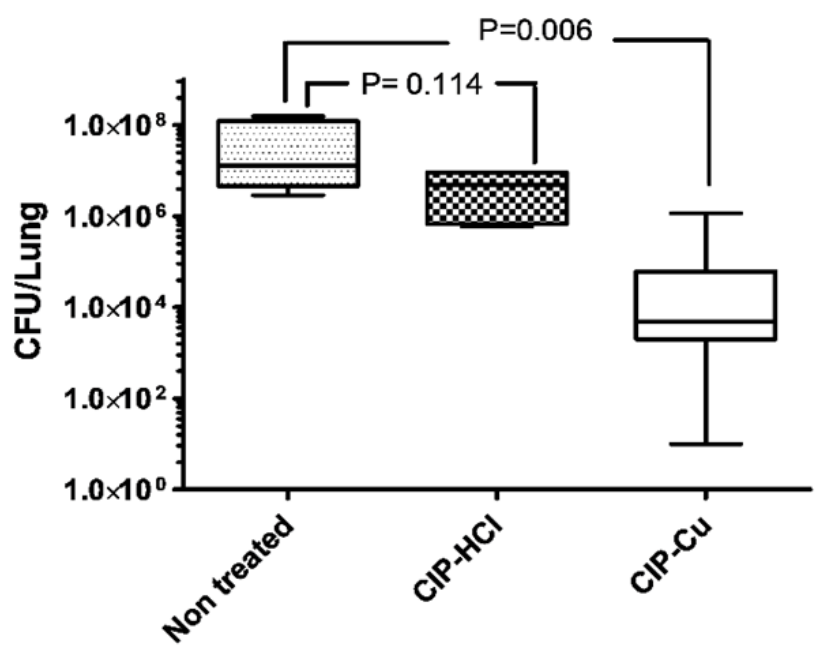

Figure 7: In vivo efficacy in a $P$. aeruginosa chronic lung infection model. Rats were infected with $6 \times 10^{6} \mathrm{CFU} / \mathrm{animal}$, then treated on days 4 and 6 with $\mathrm{CIP}-\mathrm{Cu}$ or $\mathrm{CIP}-\mathrm{HCl}$, and the lungs were harvested on day 8 . CIP dose was $1.65 \mathrm{mg} /$ animal. A) Changes in the luminescence (photons per $\mathrm{s}^{-1} \mathrm{~cm}^{-2}$ steradian $^{-1}$ ). B) Box and whisker plot of $P$. aeruginosa surviving colony forming units (CFU) per lung on day 8. Whiskers represent the minimal and maximal values $(\mathrm{n}=$ 4 for the untreated group; $n=4$ for the CIP-HCl group, and $n=7$ for the CIP-Cu group). 


\section{DISCUSSION}

\subsection{Pulmonary delivery of ciprofloxacin-copper complex significantly reduced the $P$. aeruginosa lung infection in the in vivo chronic lung infection model.}

Microparticles loaded with $\mathrm{CIP}-\mathrm{Cu}$ produced a significant reduction in bacterial lung burden compared to untreated rats (4-log reduction, Figure 7B), while the micronized powder of CIP-HCl did not change it significantly. Yet, with both powders, the total and peripheral doses of CIP delivered into the lungs of rats were identical (Figure 6). Moreover, it has been previously demonstrated that CIP or CIP-Cu solutions have the same efficacy against $P$. aeruginosa PA01 cultured either planktonically or as biofilms $[1,18]$. The better efficacy observed with the CIP$\mathrm{Cu}$ over the $\mathrm{CIP}-\mathrm{HCl}$ formulation could be attributed to a higher CIP lung residence time obtained with the CIP-Cu microparticles. Indeed, a previous study has shown that intratracheal (i.t.) administration to rats of CIP-Cu-loaded particles provides a mean AUC ratio of the CIP concentration kinetic profiles in ELF on the CIP concentration kinetic profiles in plasma 100fold higher than after administration of a CIP solution [2]. After CIP-Cu i.t. administration, CIP half-life in rat lung ELF is higher than $2 \mathrm{~h}$, while it is lower than $1 \mathrm{~h}$ after a CIP solution nebulization [2]. At the opposite, previous studies showed that CIP-HCl has poor lung targeting following oral inhalation; the drug is rapidly absorbed into the systemic circulation with a halflife of less than $1 \mathrm{~h}$ in the rat lungs, making CIP-HCl suboptimal for pulmonary delivery [5, 13, 28]. To improve the lung targeting of CIP by inhalation, Bayer AG also aimed at prolonging the residence time of CIP in the lungs. To do this, they have developed a slow-dissolving CIP formulation using the neutral (zwitterionic) solid form of the drug (CIP-betaine) instead of the faster dissolving $\mathrm{CIP}-\mathrm{HCl}$ [13]. When used as a dry powder, this neutral form of CIP enhances the efficacy of CIP in the treatment of a model of pulmonary $P$. aeruginosa infection in the rat. 
After i.t. administration at a dose of $10 \mathrm{mg} / \mathrm{kg}$, which is 2 times the total dose used in this study, a 4 to $10-\log$ reduction in $P$. aeruginosa CFU count was observed with the neutral form of CIP versus a 1.1 to $2.8-\log$ reduction for $\mathrm{CIP}-\mathrm{HCl}$ [13]. In this approach, CIP lung targeting and efficacy was improved only by controlling the dissolution rate of the CIP. Indeed, after dissolution, CIP-betaine and CIP-HCl lead to the presence of the same zwitterionic CIP in solution at the $\mathrm{pH}$ of the pulmonary fluids. In the approach presented in this study, CIP lung targeting and efficacy were improved by reducing CIP apparent permeability across the bloodlung barrier [18].

$P$. aeruginosa growing as aggregates, or biofilms, is the main responsible of the recurrent lung infections that affect cystic fibrosis patients $[29,30]$. Biofilms can be highly resistant to antibiotics compared to free swimming planktonic bacteria [31]. For example, about ten- to hundred-times more CIP is necessary to inhibit the growth of bacteria as biofilms compared to planktonic bacteria $[20,32,33]$. P. aeruginosa-entrapped in agar beads once in the lungs for several days resembles $P$. aeruginosa from biofilms observed in chronic infections $[30,34,35]$. The results of the present study indicate that complexation of CIP with $\mathrm{Cu}^{2+}$ can effectively lead to enhanced local concentration in the lung necessary to reduce $P$. aeruginosa biofilm infection.

\subsection{The in vivo chronic lung infection model with bioluminescent PA01 was highly resistant and allowed for monitoring of the progression of infection in the same animal.}

A non-invasive technique was used to monitor the progression of the pulmonary infection caused by a luminescent strain of $P$. aeruginosa (PAO1::p16Slux) entrapped in agar beads

(Figure 5). Riedel et al. demonstrated that the transformation of $P$. aeruginosa with the p16Slux system does not affect bacterial pathogenesis in rats after intranasal inoculation [23]. They also 
demonstrated that after acute PAO1::p16Slux infection, luminescence from dissected organs linearly correlated with their bacterial loads, including lungs. Animal inoculation with bacterialaden beads has been extensively used to study novel therapeutic approaches to fight chronic lung infections [36] as it resembles the lung disease observed in humans with $P$. aeruginosa chronic pneumonia with formation of pulmonary biofilm [37]. The P. aeruginosa-laden beads used in this study had a median diameter $\mathrm{D}_{50}$ of $88 \pm 3 \mu \mathrm{m}$. Growcott et al. showed that beads with a $D_{50}$ of $155 \mu \mathrm{m}$ were widely distribute in the lungs of rats, and were found as aggregates in the airways and alveolar spaces after i.t. inoculation [22]. The development of the infection in the whole lungs was confirmed in the present study by luminescence measurements from the thoracic region of the rats (Figure 5B) as well as pulmonary lesions observed after 8 days postinoculation (Figure 5C).

$P$. aeruginosa-entrapped in agar beads present in the lungs resembles $P$. aeruginosa from biofilms observed in chronic infections $[30,34,35]$. Biofilms bacterial population is heterogeneous and often harbor several subpopulations of bacteria that are resistant, persistent and/or tolerant to antimicrobial $[38,39]$. Tolerance and persistence are phenomena of increased bacterial survival in the presence of an antibiotic without an increase in the MIC [40]. Tolerant and persistent bacteria often are in dormancy. They do not grow, or very slowly, and have reduced metabolism activity when compared with growing cells [40]. To distinguish between the in-vivo antimicrobial activity of $\mathrm{CIP}-\mathrm{Cu}$ and $\mathrm{CIP}-\mathrm{HCl}$, the number of colonies appearing in 24 hours from lung homogenate was counted (Figure 7.B). This method allows the quantification mainly of the surviving bacterial population able to grow, but is not adapted for bacteria in dormancy. Similarly, luminescence is mainly emitted by metabolically active bacteria and helps to quantify bacterial proliferation [41] but also allows a more direct measurement. Riedel et al. 
[23] showed that luminescence produced by PAO1::p16Slux remained relatively stable throughout the entire growth curve, while luminescence in other species such as Listeria monocytogenes and other gram-positive bacteria decreased dramatically in stationary phase. In stationary phase, planktonic bacteria have lower metabolic activity and lower susceptibility to CIP comparable to those of certain phases of biofilm growth [42]. This suggests that, with enough sensitivity, luminescence could be used to monitor slow growing bacteria during bacterial biofilm infection. In the present study, an optimal bacteria/beads ratio was selected to inoculate the rats for achieving the best survival and a high luminescence signal (Figure 5A-B). For the two lower inoculums $\left(1.5 \times 10^{7} \mathrm{CFU}\right.$ and $\left.6 \times 10^{6} \mathrm{CFU}\right)$, after a sharp luminescence increase on day 2 , the signals decreased on day 3 to values close to these obtained on day 1 and then were stable from day 5 to day 8 . These profiles were very similar to those usually obtained by measuring the CFU numbers in the lungs $[19,21]$. However, the initial luminescence level seemed too low to accurately assess the effect of antibiotics. In addition, bacterial loads below $1.10^{7} \mathrm{CFU}$ per lung gave bioluminescence values that were close to the detection limit of the IVIS equipment. Thus, no correlation between bioluminescence and the number of CFU per lung measured on day 8 after infection could be obtained because most of the bacterial loads were less than $1.10^{7} \mathrm{CFU}$ per lung on that day (Fig. 7B). The use of a less virulent strain could help increase the inoculum used for infection and thereby increase the stable basal signal observed 4 days after infection. In addition, using a strain with a higher luminescence yield or increasing the exposure time could also increase the sensitivity of the method and make it possible to quantify the degree of infection according to the value of luminescence, not just to assess its spatial distribution. 


\subsection{Spray dried and micronized powders properties were suitable for inhalation}

The RODOS unit has long been used to characterize powders for inhalation across a range of settings on the instrument. Over the past 15 years or so, many powders of different physical and chemical properties have been analyzed by different groups revealing some consistent trends. In particular, it has been generally shown that RODOS dispersion pressures above $3 \mathrm{kPa}$ result in full dispersion and allows sizing of the primary particle sizes of a powder $[43,44]$. Similarly, dispersion pressures used in the RODOS of between 0.5-3 kPa can indicate the dispersion performance of a powder [43] agnostic to proprietary inhaler designs and even allow matching to novel inhaler dispersion performance [44]. Thus, in these preclinical in vivo feasibility studies we have characterized the powder particle size distributions to indicate how they will be dispersed as a powder and also specifically their performance for nose-only inhalation testing. Future studies will couple the powder with an inhaler system and be characterized using compendia methods to support eventual clinical testing.

\section{CONCLUSION}

Pulmonary delivery of ciprofloxacin-copper complex significantly reduced the $P$. aeruginosa in a rat chronic lung infection model. Luminescent $P$. aeruginosa entrapped in agar beads were useful to spatially monitor the development of the lung infection in rats. 


\section{REFERENCES}

[1] F. Tewes, T.F. Bahamondez-Canas, H.D.C. Smyth, Efficacy of Ciprofloxacin and Its Copper Complex against Pseudomonas aeruginosa Biofilms, AAPS PharmSciTech, (2019).

[2] B. Lamy, F. Tewes, D.R. Serrano, I. Lamarche, P. Gobin, W. Couet, A.M. Healy, S. Marchand, New aerosol formulation to control ciprofloxacin pulmonary concentration, Journal of Controlled Release, 271 (2018) 118-126.

[3] Q.T. Zhou, S.S.Y. Leung, P. Tang, T. Parumasivam, Z.H. Loh, H.-K. Chan, Inhaled formulations and pulmonary drug delivery systems for respiratory infections, Advanced drug delivery reviews, 85 (2015) 83-99.

[4] H. Stass, J. Nagelschmitz, S. Willmann, H. Delesen, A. Gupta, S. Baumann, Inhalation of a dry powder ciprofloxacin formulation in healthy subjects: a phase I study, Clinical drug investigation, 33 (2013) 419-427.

[5] H. Stass, B. Weimann, J. Nagelschmitz, C. Rolinck-Werninghaus, D. Staab, Tolerability and pharmacokinetic properties of ciprofloxacin dry powder for inhalation in patients with cystic fibrosis: a Phase I, randomized, dose-escalation study, Clinical therapeutics, 35 (2013) 15711581.

[6] A.V.L. Gontijo, J. Brillault, N. Grégoire, I. Lamarche, P. Gobin, W. Couet, S. Marchand, Biopharmaceutical characterization of nebulized antimicrobial agents in rats: 1. Ciprofloxacin, moxifloxacin, and grepafloxacin, Antimicrobial agents and chemotherapy, 58 (2014) 3942-3949.

[7] R. Endermann, H. Labischinski, C. Ladel, U. Petersen, B. Newton, Treatment of bacterial diseases of the respiratory organs, in, Bayer Pharma Aktiengesellschaft, 2011.

[8] M.C. Gaspar, N. Grégoire, J.J. Sousa, A.A. Pais, I. Lamarche, P. Gobin, J.-C. Olivier, S. Marchand, W. Couet, Pulmonary pharmacokinetics of levofloxacin in rats after aerosolization of immediate-release chitosan or sustained-release PLGA microspheres, European Journal of Pharmaceutical Sciences, 93 (2016) 184-191.

[9] D.J. Serisier, D. Bilton, A. De Soyza, P.J. Thompson, J. Kolbe, H.W. Greville, D. Cipolla, P. Bruinenberg, I. Gonda, O.-. investigators, Inhaled, dual release liposomal ciprofloxacin in noncystic fibrosis bronchiectasis (ORBIT-2): a randomised, double-blind, placebo-controlled trial, Thorax, 68 (2013) 812-817.

[10] S.N. Nurbaeti, J. Brillault, F. Tewes, J.-C. Olivier, Sustained-release microparticle dry powders of chloramphenicol palmitate or thiamphenicol palmitate prodrugs for lung delivery as aerosols, European Journal of Pharmaceutical Sciences, (2019) 105028.

[11] D. Cipolla, J. Blanchard, I. Gonda, Development of liposomal ciprofloxacin to treat lung infections, Pharmaceutics, 8 (2016) 6.

[12] N.G. Türeli, A. Torge, J. Juntke, B.C. Schwarz, N. Schneider-Daum, A.E. Türeli, C.-M. Lehr, M. Schneider, Ciprofloxacin-loaded PLGA nanoparticles against cystic fibrosis P. aeruginosa lung infections, European Journal of Pharmaceutics and Biopharmaceutics, 117 (2017) 363-371.

[13] P.J. McShane, J.G. Weers, T.E. Tarara, A. Haynes, P. Durbha, D.P. Miller, T. Mundry, E. Operschall, J.S. Elborn, Ciprofloxacin Dry Powder for Inhalation (ciprofloxacin DPI): Technical 
design and features of an efficient drug-device combination, Pulmonary Pharmacology \& Therapeutics, 50 (2018) 72-79.

[14] F. Tewes, J. Brillault, B. Lamy, P. O'Connell, J.C. Olivier, W. Couet, A.M. Healy, Ciprofloxacin-Loaded Inorganic-Organic Composite Microparticles To Treat Bacterial Lung Infection, Molecular pharmaceutics, 13 (2016) 100-112.

[15] D.C. Griffith, M.N. Dudley, M.W. Surber, K.A. Bostian, O. Rodny, Aerosol fluoroquinolone formulations for improved pharmacokinetics, in, Patents, 2014.

[16] B. Lamy, D. Remedios Serrano, P. O'Connell, W. Couet, S. Marchand, A.M. Healy, F. Tewes, Use of leucine to improve aerodynamic properties of ciprofloxacin-loaded maltose microparticles for inhalation, European Journal of Pharmaceutical Research, 1 (2019) 2-11.

[17] J. Brillault, F. Tewes, Control of the Lung Residence Time of Highly Permeable Molecules after Nebulization: Example of the Fluoroquinolones, Pharmaceutics, 12 (2020) 387.

[18] J. Brillault, F. Tewes, W. Couet, J. Olivier, In vitro biopharmaceutical evaluation of ciprofloxacin/metal cation complexes for pulmonary administration, European Journal of Pharmaceutical Sciences, 97 (2017) 92-98.

[19] I. Kukavica-Ibrulj, R. Levesque, Animal models of chronic lung infection with Pseudomonas aeruginosa: useful tools for cystic fibrosis studies, Laboratory animals, 42 (2008) 389-412.

[20] B.G.S. Torres, R. Awad, S. Marchand, W. Couet, F. Tewes, In vitro evaluation of Pseudomonas aeruginosa chronic lung infection models: Are agar and calcium-alginate beads interchangeable?, European Journal of Pharmaceutics and Biopharmaceutics, 143 (2019) 35-43.

[21] A. Bragonzi, D. Worlitzsch, G.B. Pier, P. Timpert, M. Ulrich, M. Hentzer, J.B. Andersen, M. Givskov, M. Conese, G. Döring, Nonmucoid Pseudomonas aeruginosa expresses alginate in the lungs of patients with cystic fibrosis and in a mouse model, Journal of Infectious Diseases, 192 (2005) 410-419.

[22] E. Growcott, A. Coulthard, R. Amison, E. Hardaker, V. Saxena, L. Malt, P. Jones, A. Grevot, C. Poll, C. Osborne, Characterisation of a refined rat model of respiratory infection with Pseudomonas aeruginosa and the effect of ciprofloxacin, Journal of Cystic Fibrosis, 10 (2011) 166-174.

[23] C.U. Riedel, P.G. Casey, H. Mulcahy, F. O'Gara, C.G. Gahan, C. Hill, Construction of p16Slux, a novel vector for improved bioluminescent labeling of gram-negative bacteria, Applied and environmental microbiology, 73 (2007) 7092-7095.

[24] A.D. Brunaugh, H.D. Smyth, Formulation techniques for high dose dry powders, International journal of pharmaceutics, 547 (2018) 489-498.

[25] D.J. Alexander, C.J. Collins, D.W. Coombs, I.S. Gilkison, C.J. Hardy, G. Healey, G. Karantabias, N. Johnson, A. Karlsson, J.D. Kilgour, P. McDonald, Association of Inhalation Toxicologists (AIT) Working Party Recommendation for Standard Delivered Dose Calculation and Expression in Non-Clinical Aerosol Inhalation Toxicology Studies with Pharmaceuticals, Inhalation Toxicology, 20 (2008) 1179-1189.

[26] P.J. Kuehl, T.L. Anderson, G. Candelaria, B. Gershman, K. Harlin, J.Y. Hesterman, T. Holmes, J. Hoppin, C. Lackas, J.P. Norenberg, H. Yu, J.D. McDonald, Regional particle size 
dependent deposition of inhaled aerosols in rats and mice, Inhalation Toxicology, 24 (2012) 2735 .

[27] S.R. Carvalho, A.B. Watts, J.I. Peters, S. Liu, S. Hengsawas, M.S. Escotet-Espinoza, R.O. Williams III, Characterization and pharmacokinetic analysis of crystalline versus amorphous rapamycin dry powder via pulmonary administration in rats, European Journal of Pharmaceutics and Biopharmaceutics, 88 (2014) 136-147.

[28] J. Weers, Comparison of Phospholipid-Based Particles for Sustained Release of Ciprofloxacin Following Pulmonary Administration to Bronchiectasis Patients, Pulmonary Therapy, (2019).

[29] M.L. Barclay, E.J. Begg, S.T. Chambers, P.E. Thornley, P.K. Pattemore, K. Grimwood, Adaptive resistance to tobramycin in Pseudomonas aeruginosa lung infection in cystic fibrosis, Journal of Antimicrobial Chemotherapy, 37 (1996) 1155-1164.

[30] T. Bjarnsholt, P.Ø. Jensen, M.J. Fiandaca, J. Pedersen, C.R. Hansen, C.B. Andersen, T. Pressler, M. Givskov, N. Høiby, Pseudomonas aeruginosa biofilms in the respiratory tract of cystic fibrosis patients, Pediatric pulmonology, 44 (2009) 547-558.

[31] N. Høiby, O. Ciofu, T. Bjarnsholt, Pseudomonas aeruginosa biofilms in cystic fibrosis, Future microbiology, 5 (2010) 1663-1674.

[32] M.C. Walters, F. Roe, A. Bugnicourt, M.J. Franklin, P.S. Stewart, Contributions of antibiotic penetration, oxygen limitation, and low metabolic activity to tolerance of Pseudomonas aeruginosa biofilms to ciprofloxacin and tobramycin, Antimicrobial agents and chemotherapy, 47 (2003) 317-323.

[33] H. Bandara, D. Nguyen, S. Mogarala, M. Osiñski, H. Smyth, Magnetic fields suppress Pseudomonas aeruginosa biofilms and enhance ciprofloxacin activity, Biofouling, 31 (2015) 443-457.

[34] M. Sønderholm, K.N. Kragh, K. Koren, T.H. Jakobsen, S.E. Darch, M. Alhede, P.Ø. Jensen, M. Whiteley, M. Kühl, T. Bjarnsholt, Pseudomonas aeruginosa Aggregate Formation in an Alginate Bead Model System Exhibits In Vivo-Like Characteristics, Applied and Environmental Microbiology, 83 (2017).

[35] D.P. Gnanadhas, M. Elango, A. Datey, D. Chakravortty, Chronic lung infection by Pseudomonas aeruginosa biofilm is cured by L-Methionine in combination with antibiotic therapy, Scientific reports, 5 (2015) 16043.

[36] P. Meers, M. Neville, V. Malinin, A. Scotto, G. Sardaryan, R. Kurumunda, C. Mackinson, G. James, S. Fisher, W. Perkins, Biofilm penetration, triggered release and in vivo activity of inhaled liposomal amikacin in chronic Pseudomonas aeruginosa lung infections, Journal of Antimicrobial Chemotherapy, 61 (2008) 859-868.

[37] H. Cash, D. Woods, B. McCullough, W. Johanson Jr, J. Bass, A rat model of chronic respiratory infection with Pseudomonas aeruginosa, American Review of Respiratory Disease, 119 (1979) 453-459.

[38] K. Lewis, Persister cells, Annual review of microbiology, 64 (2010) 357-372.

[39] H.-C. Flemming, J. Wingender, U. Szewzyk, P. Steinberg, S.A. Rice, S. Kjelleberg, Biofilms: an emergent form of bacterial life, Nature Reviews Microbiology, 14 (2016) 563. 
[40] N.Q. Balaban, S. Helaine, K. Lewis, M. Ackermann, B. Aldridge, D.I. Andersson, M.P. Brynildsen, D. Bumann, A. Camilli, J.J. Collins, Definitions and guidelines for research on antibiotic persistence, Nature Reviews Microbiology, 17 (2019) 441-448.

[41] S. Duncan, L.A. Glover, K. Killham, J.I. Prosser, Luminescence-based detection of activity of starved and viable but nonculturable bacteria, Appl. Environ. Microbiol., 60 (1994) 13081316.

[42] T.-F.C. Mah, G.A. O'Toole, Mechanisms of biofilm resistance to antimicrobial agents, Trends in Microbiology, 9 (2001) 34-39.

[43] S. Jaffari, B. Forbes, E. Collins, D.J. Barlow, G.P. Martin, D. Murnane, Rapid characterisation of the inherent dispersibility of respirable powders using dry dispersion laser diffraction, International journal of pharmaceutics, 447 (2013) 124-131.

[44] A.H. de Boer, P. Hagedoorn, E.M. Westerman, P.P. Le Brun, H.G. Heijerman, H.W. Frijlink, Design and in vitro performance testing of multiple air classifier technology in a new disposable inhaler concept (Twincer $($ ) for high powder doses, European journal of pharmaceutical sciences, 28 (2006) 171-178. 